\title{
SCHUR RING AND REDUCIBILITY MODULO $p$
}

\section{PEDRO MANUEL DOMINGUEZ WADE}

Department of Mathematics

Matanzas University

Cuba

e-mail: pedroalgebralineal@gmail.com

\begin{abstract}
Let $R$ be a ring of algebraic integers of an algebraic number field $F$ and let $G \leq G L_{n}(R)$ be a finite group. In [11] was proved that the $R$-span of $G$ is just the matrix ring $M_{n}(R)$ of the $n \times n$-matrices over $R$ if and only if the Brauer reduction of $R^{n}$ modulo every prime is absolutely irreducible. In this paper, we show that $\langle G\rangle_{R}=M_{n}(R)$ if and only if the Brauer reduction of $R^{n}$ modulo a finite number of primes is absolutely irreducible. Moreover, we give conditions for $n$, under which $M_{n}(R)$ is a Schur ring.
\end{abstract}

\section{Introduction}

Let $F$ be an algebraic number field with ring of algebraic integers $R$ and let $\pi=\left\{p_{1}, \ldots, p_{t}\right\}$ be a set of positive prime numbers. Assume that the $I_{i}$ are maximal ideals of $R$ such that $p_{i} \in I_{i}, i=1, \ldots, t$. Set $L_{\pi}=\left\{\frac{a}{b} \mid a, b \in R, b \notin I_{i}, i=1, \ldots, t\right\}$. Then $R_{\pi}$ denotes a localization 2010 Mathematics Subject Classification: Primary 20C20; Secondary 19A22.

Keywords and phrases: Schur ring, $\pi$-quasi-simple, $\pi$-globally simple, $G$-weight, reduction modulo $p$, reduction modulo $\pi$.

Received October 29, 2015

(C) 2015 Scientific Advances Publishers 
of $R$ at $L_{\pi}$. Thus $R_{\pi}$ is a principal ideal having quotient field of characteristic zero and containing a unique prime ideal $I_{i}$ such that $p_{i} \in I_{i}, i=1, \ldots, t$. We denote the Jacobson radical of $R_{\pi}$ be $J\left(R_{\pi}\right)$. Therefore, the residue ring $K=R_{\pi} / J\left(R_{\pi}\right)$ is a semi-simple ring of characteristic $m=\prod_{i=1}^{t} p_{i}$. Thus, we may write

$$
K=\bigoplus_{i=1}^{t} k_{i}
$$

where the $k_{i}$ are fields of characteristic $p_{i}(i=1, \ldots, t)$. Let $G$ be a finite group. Then we have

$$
K G=\bigoplus_{i=1}^{t} k_{i} G
$$

From (1.2), it follows that

$$
1=f_{1}+\cdots+f_{t},
$$

where the $f_{i}$ are orthogonal central idempotents in $K G$. Therefore,

$$
\begin{aligned}
K G & =\bigoplus_{i=1}^{t} k_{i} G \\
& =\bigoplus_{i=1}^{t} K G f_{i},
\end{aligned}
$$

with $k_{i} G=K G f_{i}$.

Now, $R_{\pi}$ is a Hausdorff space in its $J\left(R_{\pi}\right)$-topology, i.e., that $\bigcap_{j=1}^{\infty} J(R)^{j}=(0)$, so the $J\left(R_{\pi}\right)$-adic completion $\hat{R}_{\pi}$ of $R_{\pi}$ is a complete semi-local ring such that

$$
K=\hat{R}_{\pi} / J\left(\hat{R}_{\pi}\right)=R_{\pi} / J\left(R_{\pi}\right)
$$


and

$$
\hat{R}_{\pi}=\hat{R}_{\pi 1} \oplus \cdots \oplus \hat{R}_{\pi t}
$$

where the $\hat{R}_{\pi i}$ are complete local rings such that $\hat{R}_{\pi} / J\left(\hat{R}_{\pi}\right)=k_{i}$. Therefore

$$
\hat{R}_{\pi} G=\hat{R}_{\pi 1} G \oplus \cdots \oplus \hat{R}_{\pi t} G .
$$

From (1.5), we obtain

$$
1=\hat{f}_{1}+\cdots+\hat{f}_{t}
$$

where the $\hat{f}_{i}$ are orthogonal central idempotents in $\hat{R}_{\pi} G$.

In the study of the Schur subgroup of the Brauer group of a commutative ring $R$, one is interested in finding the Azumaya algebras over $R$ that are epimorphic images of a group ring $R G$ for some finite group $G$.

For a commutative ring $R$, the matrix ring $M_{n}(R)$ of the $n \times n$ matrices over $R$ is called a Schur ring if there exists a finite group $G \leq G L_{n}(R)$ such that the $R$-span of $G$ is just $M_{n}(R)$, i.e., in such case we have $\langle G\rangle_{R}=M_{n}(R)$.

Let $R$ be a ring of algebraic integers of an algebraic number field $F$ and let $G \leq G L_{n}(R)$ be a finite group. In [11], it was proved that $\langle G\rangle_{R}=M_{n}(R)$ if and only if $R^{n}$ is globally irreducible, i.e., the Brauer reduction of $R^{n}$ modulo every prime is absolutely irreducible. In this paper, we show that $\langle G\rangle_{R}=M_{n}(R)$ if and only if the Brauer reduction of $R^{n}$ modulo a finite number of primes is absolutely irreducible. Moreover, we give conditions for $n$, under which $M_{n}(R)$ is a Schur ring. 


\section{Preliminary Results}

Definition 2.1. Let $\mathbb{C}$ denote the field of complex numbers and $G \leq G L_{n}(\mathbb{C})$. We call $G$ globally irreducible if for every prime $p$ the reduction of $\mathbb{C}^{n}$ modulo $p$ is absolutely irreducible.

Let $G$ be a finite group, $p$ be a prime divisor of $|G|$, and $R$ be a complete discrete valuation ring with quotient field $F$ of characteristic 0 . We assume that the residue field $k=R / J(R)$ has characteristic $p$, where $J(R)$ denotes the Jacobson radical of $R$. With this assumption, we refer to the triple $(F ; R ; k)$ as a splitting $p$-modular system.

Recall that the Brauer reduction of a modulo for a natural prime $p$ is defined as follows. If $V$ is a $F G$-module, then there exists a full $R G$-lattice $L \subseteq V$. The $k G$-module $L / J(R) L=U$ is called a reduction of $V$ modulo $p$. Moreover, in such case, we say also that $U$ is the reduction modulo $p$ of the $R G$-lattice $L$.

According to Definition 2.1, the linear group $G$ is globally irreducible if for every prime $p$ the reduction of $\mathbb{C}^{n}$ modulo $p$ is absolutely irreducible. We know that in such case is sufficient the study of the reduction modulo $p$, for every prime $\mathrm{p}$ divisor of $|G|$. Therefore, result evident the necessity of to study the following problem:

\section{Problem 2.2.}

When the reduction modulo $p$ of the an absolutely irreducible $F G$-module $V$ is an absolutely irreducible $k G$-module, being $k$ a field of characteristic $p$ ?

\section{Some Properties of Certain Induced Modules}

Let $G$ be a finite group with splitting field $k$, and let $Q$ be a $p$-subgroup of $G$. Assume that $s=|G: Q|$ and let $X^{+}=\left\{x_{1}, \ldots, x_{s}\right\}$ be a full set of representatives in $G$ of the cosets in $G / Q$. Then $\operatorname{Ind}_{Q}^{G}(k)$ is isomorphic to $k G Q^{+}$as left $k G$-module, where $\mathrm{Q}^{+}=\left\{\sum_{x \in X^{+}} \alpha x \in k G\right\}$. 
Set $X=\left\{x_{i}-x_{i} y, y \in Q\right\}$. We denote the left ideal generated by $X$ in $k G$ by $I_{Q}(G)$. We claim that

$$
\begin{aligned}
\operatorname{rank}_{k}\left(I_{Q}(G)\right) & =|G: Q|(|Q|-1) \\
& =|G: P| \frac{|P|}{|Q|}(|Q|-1) .
\end{aligned}
$$

Thus, we have

$$
k G / I_{Q}(G) \cong k G Q^{+}
$$

as $k$-modules. We now assume that $\mathrm{Q}<\mathrm{Q}^{\prime}$, where $\mathrm{Q}^{\prime}$ is also a $p$-subgroup of $G$. Set $\mathrm{X}_{\mathrm{Q}}^{\mathrm{Q}^{\prime}}=\left\{x_{i}-x_{j}, x_{j}=y x y^{\prime}, y \in Q\right.$ and $\left.y^{\prime} \in Q^{\prime}\right\}$. Then $k G / I_{Q}(G)$ contains a left ideal isomorphic to the left ideal generated by $\mathrm{X}_{\mathrm{Q}}^{\mathrm{Q}^{\prime}}$. We denote this ideal by $I_{\mathrm{Q}}^{\mathrm{Q}^{\prime}}$. Observe that

$$
\operatorname{rank}_{k}\left(I_{\mathrm{Q}}^{\mathrm{Q}^{\prime}}\right)=|G: P| \frac{|P|}{\left|Q^{\prime}\right|}\left(\frac{\left|Q^{\prime}\right|}{|Q|}-1\right) .
$$

Let us write $T_{Q}$ by $k G / I_{Q}(G)$. Thus we have

$$
T_{Q} / I_{\mathrm{Q}}^{\mathrm{Q}^{\prime}} \cong k G Q^{\prime+} \text {. }
$$

It is well know that

$$
k G=\bigoplus_{j=1}^{r} P_{S_{j}}^{\operatorname{dim}} S_{j}
$$

where $P_{S_{j}}$ is the projective cover of the simple $k G$-module $S_{j}$ and $r$ is the number of conjugacy classes of $p$-regular elements of $G$.

From (3.9), the following holds:

$$
k G Q^{+}=\bigoplus_{j=1}^{r} M_{j}^{Q}
$$

where $M_{j}^{Q}=P_{S_{j}}^{\operatorname{dim} S_{j}} / P_{S_{j}}^{\operatorname{dim} S_{j}} I_{Q}(G)$. 
The following two lemmas are easy but useful to our results:

Lemma 3.3. Let $G$ be a finite group with splitting field $k$ of characteristic $p$. Fixed $P \in S y l_{p}(G)$. Then $I_{P}(G)$ is an annihilator of the trivial kG-module.

Proof. Since for all finite group $G$, the trivial module is a $k G / I_{P}(G)$ -module, the assertion follows.

Lemma 3.4. Let $G$ be a finite group with splitting field $k$ of characteristic $p$. Fixed $P \in S y l_{p}(G)$. We denote the Jacobson radical of $k G$ by $J(G)$. Then $J(G) \subseteq I_{P}(G)$ if and only if $k G / I_{P}(G)$ is semisimple.

Proof. Since every indecomposable $k G$-module, direct summand of $k G / I_{P}(G)$, is annihilated by $I_{P}(G)$ the result follows.

The converse implication is trivial.

Lemma 3.5. Let $G$ be a finite group with splitting field $k$ of characteristic $p$, and let $S$ be a simple $k G$-module. Set $P \in S y l_{p}(G)$ fixed. Then $M_{j}^{P}$ is a projective $k G$-module if and only if $P_{S_{j}}$ is a blocks of defect zero.

Proof. Let $J(G)$ be the Jacobson radical of $k G$. We to check two cases.

Case 1. $J(G) \subseteq I_{P}(G)$.

In this case, the assertion follows by Lemma 3.4.

Case 2. $J(G) \nsubseteq I_{P}(G)$.

Assume that $M_{j}^{Q} \cong P_{S_{j}}^{l}$ is a projective $k G$-module, where $l$ is the multiplicity of $P_{S_{j}}$ as direct summand of $M_{j}^{Q}$. We show that $P_{S_{j}}$ is a simple $k G$-module. 
Since $I_{P}(G)$ is left ideal of $k G$ from (3.9) is follows that

$$
I_{P}(G)=P_{S_{1}}^{\operatorname{dim} S_{1}} I_{P}(G) \oplus \cdots \oplus P_{S_{r}}^{\operatorname{dim} S_{r}} I_{P}(G) .
$$

We have $P_{S_{j}}^{l} I_{P}(G)=0$ by assumption, so we deduce that $P_{S_{j}}^{\operatorname{dim} S_{j}} I_{P}(G)$ is a projective $k G$-module, where the multiplicity of $P_{S_{j}}$ is equal to $\operatorname{dim}\left(S_{j}\right)-l$, i.e., we have

$$
P_{S_{j}}^{\operatorname{dim} S_{j}} I_{P}(G)=P_{S_{j}}^{\operatorname{dim}\left(S_{j}\right)-l}
$$

Therefore, we may assert that $P_{S_{j}} I_{P}(G)$ is a right indecomposable $I_{P}(G)$-module such that

$$
\left(P_{S_{j}} I_{P}(G)\right)^{\operatorname{dim} S_{j}}=P_{S_{j}}^{\operatorname{dim}\left(S_{j}\right)-l} .
$$

We assume that $\alpha=\operatorname{dim}\left(P_{S_{j}} I_{P}(G)\right)$ and $\beta=\operatorname{dim}\left(P_{S_{j}}\right)$. According to (3.12), we way write the following equality:

$$
\alpha \operatorname{dim}\left(S_{j}\right)=\beta\left(\operatorname{dim}\left(S_{j}\right)-l\right) .
$$

From (3.13), it follows that

$$
\frac{\alpha}{\operatorname{dim}\left(S_{j}\right)-l}=\frac{\beta}{\operatorname{dim} S_{j}}
$$

We now claim that the Equality (3.14) is true if and only if $\frac{\alpha}{\operatorname{dim}\left(S_{j}\right)-l}$ $=\frac{\beta}{\operatorname{dim} S_{j}}=1$. Thus, the following holds $\operatorname{dim} S_{j}=\operatorname{dim} P_{S_{j}}$, which is what we need to prove.

Conversely, by assumption it follows that

$$
P_{S_{j}}^{\operatorname{dim} S_{j}} I_{P}(G)=\left(P_{S_{j}} I_{P}(G)\right)^{\operatorname{dim} S_{j}}
$$


where $\operatorname{dim}\left(P_{S_{j}} I_{P}(G)\right)=\operatorname{dim}\left(S_{j}\right)-l$ with $l=\operatorname{dim} S_{j p^{\prime}}, \quad$ being $\operatorname{dim} S_{j p^{\prime}}$ the $p^{\prime}$-part of $\operatorname{dim} S_{j}$. Thus, we deduce that $P_{S_{j}}^{\operatorname{dim} S_{j}} I_{P}(G)=P_{S_{j}}^{\operatorname{dim}\left(S_{j}\right)-l}$.

So we are done.

Lemma 3.6. Let $G$ be a finite group with splitting field $k$ of characteristic $p$ and let $P \in S_{y l}(G)$ fixed. Then every indecomposable $k G$-module direct summand of $k G P^{+}$has a radical vertex.

Proof. Let $N_{G}(P)$ be the normalizer of $P$. According to the Green correspondence, every indecomposable $k G$-module direct summand of $k G P^{+} \cong \operatorname{Ind}_{P}^{N_{G}(P)} \operatorname{Ind}_{N_{G}(P)}^{G}(k)$ has vertex $P$ or a vertex in $P \bigcap P^{g}$, $g \in G-N_{G}(P)$. Observe that if $P$ is a normal subgroup of $G$, then $k G P^{+}$ is semi-simple, so every indecomposable $k G$-module direct summand of $k G P^{+}$is a simple $k P$-module with vertex $P$. Therefore, we now consider the case where $P$ is not a normal subgroup of $G$. Assume that $U$ is an indecomposable $k G$-module with vertex $Q \leq P$, being $U$ a direct summand of $k G P^{+}$. We to check two cases.

- Case 1. $Q=1$ or $Q=P$.

The assertion results trivially by assumption.

- Case 2. $1<Q<P$.

In this case $Q=P \bigcap P^{g}$, for some $g \in G-N_{G}(P)$. Let $N_{P}(Q)$ be the normalizer of $Q$ in the Sylow $p$-subgroup $P$. Since $P \bigcap N_{G}(Q)=N_{P}(Q)$ and $P^{g} \bigcap N_{G}(Q)=N_{P}^{g}(Q)$ are Sylow p-subgroups of $N_{G}(Q)$, we deduce that $g \in N_{G}(Q)-N_{P}(Q)$. We now shows that $N_{P}(Q)$ is not a normal subgroup of $N_{G}(Q)$. 
Let us write $\mathbb{P}$ for $N_{P}(Q)$. Conversely, we assume that $\mathbb{P}$ is a normal subgroup of $N_{G}(Q)$. In such case $\mathbb{P}=P \bigcap P^{g}=Q$, which is a contradiction. Now, since $Q=\mathbb{P} \bigcap \mathbb{P}^{g}$ is follows that

$$
Q \supseteq O_{p}\left(N_{G}(Q)\right) .
$$

But on the other hand, $Q$ is a normal $p$-subgroup of $N_{G}(Q)$, and so is contained in $O_{p}\left(N_{G}(Q)\right)$. Thus we have equality.

Many of the properties of the $k G$-modules with trivial source was studied by several authors. In particular, Okuyama's obtained the following results (see [9]).

Lemma 3.7. Let $S$ be a simple $k G$-module with vertex $Q$ and trivial source. Then the Green correspondent $f(S)$ of $S$ is a simple projective $k\left[N_{G}(Q) / Q\right]$-module.

Lemma 3.8. Let $S$ be a simple $k G$-module with vertex $Q$ and trivial source. Then the p-part of $\operatorname{dim} S$ is equal to $\frac{|P|}{|Q|}$, where $P \in S y l_{p}(G)$.

Alperin's obtained the following result (see [1]):

Lemma 3.9. Let $P$ be a Sylow p-subgroup of $G$. If $W$ is a simple projective $k\left[N_{G}(Q) / Q\right]$-module, then its Green correspondent of $W$ is isomorphic to an indecomposable direct summand of $k G P^{+}$.

\section{G-Weight and Main Proprieties}

In the rest of this paper, we will assume the notations and terminologies used in the last section. 
Lemma 4.10. Let $G$ be a finite group, $k$ be a splitting field for $G$, and $Q \neq 1$ be a radical p-subgroup of $G$. If $M_{j}^{Q}$ has an indecomposable nonprojective $k G$-module as direct summand, then it is unique, up it isomorphism.

Proof. By the Krull-Schmidt theorem, each left $k G$-module $M_{j}^{Q}$ can be decomposed of unique manner as a direct sum of indecomposable $k G$-modules, i.e., we may write

$$
M_{j}^{Q}=\bigoplus_{\gamma=1}^{\mu} U_{\gamma}
$$

where the $U_{\gamma}$ are indecomposable $k G$-modules.

We now assume that $U_{\gamma}$ is direct summand in (4.16), which is an indecomposable non-projective $k G$-module. Firstly, we show that $P_{S_{j}}$ is the projective cover of $U_{\gamma}$.

Since $P_{S_{j}} / \operatorname{Rad}\left(P_{S_{j}}\right) \cong U_{\gamma} / \operatorname{Rad}\left(U_{\gamma}\right) \cong S_{j}$, we deduce that there is an epimorphism $P_{S_{j}} \rightarrow U_{\gamma}$, which necessarily is essential by Nakayama's lemma.

We now show that $U_{\gamma}$ is unique, up to isomorphism.

Suppose that $U_{\gamma^{\prime}}$ is other indecomposable non-projective $k G$-module in the decomposition (4.16). Since $P_{S_{j}}$ is projective cover of $U_{\gamma}$ and $U_{\gamma^{\prime}}$ we assert that there are two essential epimorphisms $\theta_{1}: P_{S_{j}} \rightarrow U_{\gamma}$ and $\theta_{2}: P_{S_{j}} \rightarrow U_{\gamma^{\prime}}$. We define the epimorphism $\sigma: U_{\gamma} \rightarrow U_{\gamma^{\prime}}$ given by $\sigma\left(\theta_{1}(a)\right)=\theta_{2}(a), a \in P_{S_{j}}$. Let $\Omega\left(U_{\gamma}\right)$ and $\Omega\left(U_{\gamma^{\prime}}\right)$ be the Heller operators of $U_{\gamma}$ and $U_{\gamma^{\prime}}$, respectively. Then we may write

$$
\operatorname{ker} \sigma=\left\{\theta_{1}(a) \in U_{\gamma}: a \in \Omega\left(U_{\gamma^{\prime}}\right)\right\}
$$


Thus we may assert that $\operatorname{ker} \sigma \cong \Omega\left(U_{\gamma^{\prime}}\right)$ by assumption. Hence, we have

$$
P_{S_{j}} / \Omega\left(U_{\gamma^{\prime}}\right) \cong U_{\gamma} / \text { ker } \sigma \cong U_{\gamma^{\prime}}
$$

We claim that the relation (4.17) is true if and only if $P_{S_{j}} \cong U_{\gamma}$ or ker $\sigma=0$. By assumption, we may deduce that the unique case possible is ker $\sigma=0$. Therefore $U_{\gamma} \cong U_{\gamma^{\prime}}$, which is what we need to prove.

According to the Lemmas 3.5 and 4.10 takes place the following definition:

Definition 4.11. A $G$-weight for $G$ is a pair $(U, Q)$, where $U$ is a direct summand of $k G P^{+}$with vertex $Q$, which is simple or an indecomposable non-projective $k G$-module.

The following theorem is fundamental in our investigation.

Theorem 4.12. Let $G$ be a finite group with splitting field $k$ of characteristic $p$. Then the number of non-isomorphic G-weights equals the number of conjugacy classes of p-regular elements of $G$.

Proof. From (3.10), we have

$$
k G P^{+}=\bigoplus_{j=1}^{r} M_{j}^{P}
$$

where $r$ is the number of conjugacy classes of $p$-regular elements of $G$ and the $M_{j}^{P}$ are left $k G$-modules such that

$$
M_{j}^{P} \cong P_{S_{j}}^{\operatorname{dim} S_{j}} / P_{S_{j}}^{\operatorname{dim} S_{j}} I_{P}(G)
$$

for some simple $k G$-module $S_{j}$.

We check two cases.

Case 1. $M_{j}^{P}$ is projective.

In such case we have $M_{j}^{P}=\oplus P_{S_{j}}$. According to Lemma 3.5, we obtain $P_{S_{j}} \cong S_{j}$. Therefore, the assertion follows by assumption. 
Case 2. $M_{j}^{P}$ is not projective.

If $M_{j}^{P}$ is not projective, then has a unique indecomposable nonprojective $k G$-module as direct summand, by Lemma 4.10. Thus the result follows by assumption.

\section{Conditions for the Reducibility Modulo $p$ of an Irreducible Brauer $p$-Character}

Let $G$ be a finite group, $p$ be a prime divisor of $|G|$, and $R$ be a complete discrete valuation ring with quotient field $F$ of characteristic 0 . We assume that the residue field $k=R / J(R)$ has characteristic $p$, where $J(R)$ denotes the Jacobson radical of $R$. With this assumption, we refer to the triple $(F ; R ; k)$ as a splitting $p$-modular system.

Lemma 5.13. Let $G$ be a finite group and $k$ be a splitting field for $G$. Let $U_{1}, \ldots, U_{r}$ be a complete list of non-isomorphic G-weights, with projective covers $P_{S_{1}}, \ldots, P_{S_{r}}$, respectively. Then the Brauer characters $\phi_{U_{1}}, \ldots, \phi_{U_{r}}$ of the $G$-weights form a basis in the space $\mathbb{C}^{p-r e g(G)}$ of class functions on the p-regular elements of $G$.

Proof. Everything follows from the formula

$$
\tau=\left\langle\phi_{P_{S_{i}}}, \phi_{U_{j}}\right\rangle= \begin{cases}\tau=0, & \text { if } i \neq j ; \\ \tau=1, & \text { if } i=j \text { and } U_{j} \cong S_{i} ; \\ \tau>1, & \text { if } i=j \text { and } U_{j} \cong S_{i},\end{cases}
$$

and the fact that the number of non-isomorphic $G$-weights modules equals the number of $p$-regular conjugacy classes of $G$. Thus if $\sum_{i=1}^{r} \lambda_{i} \phi_{U_{i}}=0$, we have $\left\langle\phi_{P_{S_{i}}}, \phi_{U_{i}}\right\rangle \lambda_{i}=0$, so $\lambda_{i}=0$, which shows that the are independent, and hence form a basis. 
Theorem 5.14. Let $(F ; R ; k)$ be a splitting $p$-modular system for the finite group $G$. The simple $k G$-module $S$ is the reduction modulo $p$ of an $R G$-lattice if and only if $S$ is a G-weight.

Proof. Let $S$ be a simple $k G$-module with projective cover $P_{S}$, and let $U_{i}$ be a $G$-weight such that $U_{i} / \operatorname{Rad}\left(U_{i}\right) \cong S$. Assume that $S$ is the reduction modulo $p$ of an $R G$-lattice. According to the Lemma 5.13, we may write

$$
\sum_{i=1}^{r} \lambda_{i} \phi_{U_{i}}=\phi_{S} .
$$

From (5.19), we may write

$$
\left\langle\phi_{S}, \phi_{U_{i}}\right\rangle \lambda_{i}=\left\langle\phi_{S}, \phi_{S}\right\rangle
$$

Since $S$ and $U_{i}$ are liftable to one $R G$-lattice, and $S$ is the radical quotient of $U_{i}$, it follows that $\left\langle\phi_{S}, \phi_{U_{i}}\right\rangle=\left\langle\phi_{S}, \phi_{S}\right\rangle$, so $\lambda_{i}=1$.

Conversely, since $k G P^{+}$is the reduction modulo $p$ of the $R G$-lattice $R G P^{+}$the result follows.

Theorem 5.15. Let $G$ be a finite group with $P \in S y l_{p}(G)$ fixed, and let $S$ be a simple $k G$-module with radical vertex $Q$ and trivial source $k$. Then $S$ is a G-weight.

Proof. Combining the Lemmas 3.7 and 3.9, we deduce that $S$ is a direct summand of $k G P^{+}$. Therefore, by assumption, the assertion follows.

Combining the Theorems 5.14 and 5.15, we deduce the following result:

Corollary 5.16. Let $(F ; R ; k)$ be a splitting $p$-modular system for the finite group $G$. The simple $k G$-module $S$ has trivial source if and only if $S$ is the reduction modulo $p$ of an RG-lattice. 


\section{Schur Ring and Globally Simple Modules}

Definition 6.17. Let $R$ be a commutative ring and let $G \leq G L_{n}(R)$ be a finite group. Then the matrix ring $M_{n}(R)$ is called Schur ring (more briefly $S$-ring) if $\langle G\rangle_{R}=M_{n}(R)$.

Lemma 6.18. Let $k$ be a field of characteristic $p$ and let $G \leq G L_{n}(k)$ be a finite group with splitting field $k$. Let us write $U$ for $k^{n}$. Then $U$ is a simple $k G$-module if and only if $\langle G\rangle_{k}=M_{n}(k)$.

Proof. If $U$ is a simple $k G$-module, then the assertion follows by Burnside's theorem. Conversely, since $U^{n} \cong M_{n}(k)$ the result follows by assumption.

Let $G$ be a finite group, $\pi=\left\{p_{1}, \ldots, p_{t}\right\}$ be a finite set of prime numbers and $R_{\pi}$ be a localization of $R$ at $L_{\pi}$, where $R$ is the ring of integers of $F$, being $F$ the quotient field of $R$ and splitting field of $G$. We assume that the residue field $K=R_{\pi} / J\left(R_{\pi}\right)$ has characteristic $m=\prod_{i=1}^{t} p_{i}$, where $J(R)$ denotes the Jacobson radical of $R_{\pi}$. With this assumption, we refer to the triple $(F ; R ; K)$ as a splitting $\pi$-modular system.

The reduction modulo $\pi$ of an $R G$-module is defined as follows. If $V$ is a $R G$-module, then there exists a full $R_{\pi} G$-lattice $\mathfrak{L} \subseteq V$. The $K G$-module $\mathfrak{L} / J(R) \mathfrak{L}=U$ is called a reduction of $V$ modulo $\pi$. Moreover, in such case, we say also that $U$ is the reduction modulo $\pi$ of the $R_{\pi} G$-lattice $\mathfrak{L}$.

Let $G \leq G L_{n}(R)$ be a finite group. We consider the natural projection $\sigma: G L_{n}\left(R_{\pi}\right) \rightarrow G L_{n}(K)$. Then $\sigma(G)=\bar{G}$ is called reduction of $G$ modulo $\pi$. 
In such case, we way write

$$
\bar{G}=\bar{G}_{1} \otimes \cdots \otimes \bar{G}_{t},
$$

where $\bar{G}_{i}(i=1, \ldots, t)$ is the reduction of $G$ modulo $p_{i}$.

Definition 6.19. Let $(F, R, K)$ be an $m$-modular system, where $\pi=\left\{p_{1}, \ldots, p_{t}\right\}$ is a set of prime numbers. Assume that $G \leq G L_{n}(R)$ is a finite group. Let us write $V$ for $R^{n}$ and we write $U$ for $K^{n}$. Then $V$ is called $\pi$-quasi-simple if each direct summand $U f_{i}$ is a simple $\bar{G}_{i}$-weight.

Lemma 6.20. Let $(F, R, K)$ be an m-modular system, and let $G \leq G L_{n}(R)$ be a finite group. Let us write $V$ for $R^{n}$ and we write $U$ for $K^{n}$. Then $\langle\bar{G}\rangle_{K}=M_{n}(K)$ if and only if $V$ is $\pi$-quasi-simple.

Proof. We have

$$
\begin{aligned}
\langle\bar{G}\rangle_{K} & =\langle\bar{G}\rangle_{K} f_{1} \oplus \cdots \oplus\langle\bar{G}\rangle_{K} f_{t} \\
& =\left\langle\bar{G}_{1}\right\rangle_{k_{1}} \oplus \cdots \oplus\left\langle\overline{G_{t}}\right\rangle_{k_{t}} \\
& =M_{n}\left(k_{1}\right) \oplus \cdots \oplus M_{n}\left(k_{t}\right) \\
& =M_{n}(K) f_{1} \oplus \cdots \oplus M_{n}(K) f_{t} \\
& =M_{n}(K) .
\end{aligned}
$$

From (6.21), it follows that $\left\langle\bar{G}_{i}\right\rangle_{k_{i}}=M_{n}\left(k_{i}\right)$ for all $i(1 \leq i \leq t)$. Therefore, applying the last lemma we assert that $U f_{i}$ is a simple $k_{i} \bar{G}_{i}$-module for every $i$. Hence, by Theorem 5.14, the result follows. On the other hand, by assumption and applying again the Lemma 6.18, we deduce that $\left\langle\bar{G}_{i}\right\rangle_{k_{i}}=M_{n}\left(k_{i}\right)(i=1, \ldots, t)$. Therefore, the equality follows. 
Definition 6.21. Let $(F, R, K)$ be a $m$-modular system, and let $\left.G \leq G L_{n}\left(R_{\pi}\right)\right)$ be a finite group. Assume that $\pi$ is the set of the positive prime divisors of $|G|$. If $R^{n}$ is a $\pi$-quasi-simple, then we say that $G$ is $\pi$-globally simple.

Lemma 6.22. Let $(F, R, K)$ be an m-modular system, and let $G \leq G L_{n}(R)$ be a finite group. Assume that $\pi$ is a set of the prime divisors of $|G|$. Then $\langle G\rangle_{R_{\pi}}=M_{n}\left(R_{\pi}\right)$ if and only if $G$ is $\pi$-globally simple.

Proof. By assumption, we may write

$$
\langle\bar{G}\rangle_{K}=M_{n}(K)
$$

Therefore, we may assert that $R^{n}$ is $\pi$-quasi-simple by Lemma 6.20. Thus, by assumption, the result follows. On the other hand, by assumption and applying the Lemma 6.18 , we obtain

$$
\langle\bar{G}\rangle_{K}=M_{n}(K)
$$

From (6.23), it follows that

$$
R_{\pi} \otimes_{K}\langle\bar{G}\rangle_{K}=R_{\pi} \otimes_{K} M_{n}(K)
$$

Since $\langle G\rangle_{R_{\pi}} \cong R_{\pi} \otimes_{K}\langle\bar{G}\rangle_{K}$ and $M_{n}\left(R_{\pi}\right) \cong R_{\pi} \otimes_{K} M_{n}(K)$ the assertion follows.

Theorem 6.23. Let $R$ be a ring of algebraic integers and let $G \leq G L_{n}(R)$ be a finite group. Then $\langle G\rangle_{R}=M_{n}(R)$ if and only if $G$ is $\pi$-globally simple.

Proof. From $\langle G\rangle_{R}=M_{n}(R)$, it follows that $\langle G\rangle_{R_{\pi}}=M_{n}\left(R_{\pi}\right)$. Hence, the result follows by Lemma 6.22. Conversely, according to the Lemma 6.22, we have

$$
\langle G\rangle_{R_{\pi}}=M_{n}\left(R_{\pi}\right)
$$

Therefore, the assertion follows by assumption. 
Theorem 6.24. Let $R$ be a ring of algebraic integers with quotient field $F$ and let $G \leq G L_{n}(R)$ be a finite group with splitting field $F$. Assume that $\pi=\left\{p_{1}, \ldots, p_{t}\right\}$ is the set of prime divisors of $|G|$ and we denote the $\pi$-part of $n$ by $n_{\pi}$. If $\langle G\rangle_{R}=M_{n}(R)$, then $n_{\pi}=\prod_{i=1}^{t} \mid \frac{\left|P_{i}\right|}{\left|Q_{i}\right|}$, where $P_{i} \in S y l_{p_{i}}(G)$ and $Q_{i}$ is the vertex of the $k_{i} G$-module $K^{n} f_{i}$.

Proof. According to Theorem 6.23, each $K^{n} f_{i}$ is a simple $G$-weight. Hence, the $p_{i}$-part of $\operatorname{dim} K^{n} f_{i}$ is $\frac{\left|P_{i}\right|}{\left|Q_{i}\right|}$ by Lemma 3.8. Since $n=\operatorname{dim}$ $R^{n}=\operatorname{dim} K^{n}=\operatorname{dim} K^{n} f_{i}(i=1, \ldots, t)$ the assertion follows.

Theorem 6.25. Let $R$ be a ring of algebraic integers with quotient field $F$ and let $G$ be a finite group with splitting field $F$. Assume that $\pi=\left\{p_{1}, \ldots, p_{t}\right\}$ is the set of prime divisors of $|G|$, and $H$ is a $\pi$-subgroup of $G$ such that $N_{G}\left(Q_{i}\right) \leq H$, where $Q_{i} \in S y l_{p_{i}}(H)(i=1, \ldots, t)$. If $\operatorname{Ind}_{H}^{G}(R)$ is a simple FG-module, then $M_{n}(R)$ is an $S$-ring, for $n=\frac{|G|}{|H|}$.

Proof. Let $U$ be the reduction modulo $\pi$ of $\operatorname{Ind}_{H}^{G}(R)$. Since $Q_{i}$ is the vertex of the trivial $k_{i} H$-module we deduce that $\operatorname{Ind}_{H}^{G}\left(k_{i}\right) \cong U f_{i}$ has vertex $Q_{i}$ and trivial source. Therefore, we may assert that $U f_{i}$ is a $G_{i}$-weight for all $i=1, \ldots, t$. Since $U f_{i}(i=1, \ldots, t)$ is the reduction modulo $p_{i}$ of the simple FG-module $\operatorname{Ind}_{H}^{G}(R)$ we deduce that $U f_{i}$ is a simple $k_{i} G$-module. Therefore, by assumption, we may assert that $\operatorname{Ind} d_{H}^{G}(R)$ is $\pi$-globally simple. Since $\operatorname{dim} \operatorname{Ind} d_{H}^{G}(R)=\frac{|G|}{|H|}$ the assertion follows by Theorem 6.23. 


\section{References}

[1] J. L. Alperin, Weights for finite groups, Proceedings of Symposia in Pure Mathematics 47 (1987), 369-379.

[2] P. Huu Tiep, Globally irreducible representations of the finite symplectic group Sp4(q), Comm. in Algebra 22 (1994), 6439-6457.

[3] P. Huu Tiep, Basic spin representations of $2 S_{n}$ and $2 A_{n}$ as globally irreducible representations, Archive Math. 64 (1995), 103-112.

[4] P. Huu Tiep, Weil representations as globally irreducible representations, Math. Nachr. 184 (1997), 313-327.

[5] P. Huu Tiep, Globally irreducible representations of finite groups and integral lattices, Geomet. Dedicata 64 (1997), 85-123.

[6] I. M. Isaacs, Characters of pi-separable groups, J. Algebra 86 (1984), 98-128.

[7] G. James and M. W. Liebeck, Representations and Characters of Groups, Cambridge University Press, Cambridge, 2001.

[8] G. Navarro, Characters and Blocks of Finite Groups, Cambridge University Press, New York, 1998.

[9] T. Okuyama, Module correspondence in finite groups, Hokkaido Mathematical Journal 10 (1981), 299-318.

[10] G. R. Robinson, R. Geoffrey and R. Staszewski, On the representation theory of $\pi$-separable groups, J. Algebra 119(1) (1988), 226-232.

[11] A. E. Zalesskii and F. Van Oystaeyen, Finite groups over arithmetical rings and globally irreducible representations, J. Algebra 215 (1999), 418-436.

[12] O. Zariski and P. Samuel, Commutative Algebra (1958). 